\title{
Polarization amplitudes in $\tau^{-} \rightarrow \nu_{\tau} V P$ decay beyond the Standard Model
}

\author{
L. R. Dai ${ }^{1,2, *}$ and E. Oset ${ }^{2, \dagger}$ \\ ${ }^{1}$ Department of Physics, Liaoning Normal University, Dalian 116029, China \\ ${ }^{2}$ Departamento de Física Teórica and IFIC, \\ Centro Mixto Universidad de Valencia-CSIC, \\ Institutos de Investigacíon de Paterna, \\ Aptdo. 22085, 46071 Valencia, Spain
}

(Dated: November 20, 2018)

\begin{abstract}
We study the amplitudes of $\tau^{-} \rightarrow \nu_{\tau} V P$ decay for the different polarizations of the vector meson $V$, using a formalism where the mapping from the quark degrees of freedom to the meson ones is done with the ${ }^{3} P_{0}$ model. We extend the formalism to a case, with the operator $\gamma^{\mu}-\alpha \gamma^{\mu} \gamma_{5}$, that can account for different models beyond the Standard Model and study in detail the $\tau^{-} \rightarrow \nu_{\tau} K^{* 0} K^{-}$ reaction for the different polarizations of the $K^{* 0}$. The results are shown in terms of the $\alpha$ parameter that differs for each model. We find that $\frac{d \Gamma}{d M_{\mathrm{inv}}^{\left(K^{* 0} K^{-}\right)}}$is very different for each of third components of the vector spin, $M= \pm 1,0$, and in particular the magnitude $\left.\frac{d \Gamma}{d M_{\mathrm{inv}}^{\left(K^{* 0} K^{-}\right)}}\right|_{M=+1}-\left.\frac{d \Gamma}{d M_{\mathrm{inv}}^{\left(K^{* 0} K^{-}\right)}}\right|_{M=-1}$ is very sensitive to the $\alpha$ parameter, which makes the investigation of this magnitude very useful to test different models beyond the standard model.
\end{abstract}

\footnotetext{
*dailr@lnnu.edu.cn

$\dagger$ oset@ific.uv.es
} 


\section{INTRODUCTION}

The $\tau$ decays have received much attention through the years and have proved to be a good source of information on weak interaction, and also strong interaction from the hadronic decay modes [1-7]. While most of the attention in hadronic decays is given to the modes $\tau^{-} \rightarrow \nu_{\tau} M, \tau^{-} \rightarrow \nu_{\tau} M_{1} M_{2}$, with $M_{1} M_{2}$ pseudoscalar mesons, much less is known about $\tau^{-} \rightarrow \nu_{\tau} V M$ decays, with $V$ a vector and $M$ a pseudoscalar, although several modes are measured, $\tau^{-} \rightarrow \nu_{\tau} \omega \pi^{-}[8,9], \tau^{-} \rightarrow \nu_{\tau} K^{* 0} K^{-}[10], \tau^{-} \rightarrow \nu_{\tau} K^{*-} \eta[11], \tau^{-} \rightarrow \nu_{\tau} \omega K^{-}$ [12], $\tau^{-} \rightarrow \nu_{\tau} \rho^{-} \pi^{0}$ [13], $\tau^{-} \rightarrow \nu_{\tau} K^{* 0} \pi^{-}$[10], $\tau^{-} \rightarrow \nu_{\tau} \phi \pi^{-}$[14]. Theoretically there have been also a few works dealing with these reactions. The $\tau^{-} \rightarrow \nu_{\tau} \rho \eta$ decay is studied in [15] using the extended the Nambu-Jona-Lasinio model. The $\tau^{-} \rightarrow \nu_{\tau} K^{*} \bar{K}$ decay is evaluated in [6] using vector meson dominance. The $\tau^{-} \rightarrow \nu_{\tau} \omega \pi^{-}$decay is also addressed in [6, 7] and in $[16,17]$. In Ref. [6, 7] the $\tau^{-} \rightarrow \nu_{\tau}\left(\rho^{-} \bar{K}^{0}+\rho^{0} K^{-}\right)$mode is also investigated, together with the $\tau^{-} \rightarrow \nu_{\tau} \omega K^{-}$and $\tau^{-} \rightarrow \nu_{\tau} K^{*} \eta$ modes. The widths are obtained summing over the polarizations of the vector meson.

A different approach to the $\tau^{-} \rightarrow \nu_{\tau} M_{1} M_{2}, \tau^{-} \rightarrow \nu_{\tau} V M, \tau^{-} \rightarrow \nu_{\tau} V V$ reactions is done in [18] where the $\tau^{-} \rightarrow \nu_{\tau} \bar{u} d$ ( $\bar{u} s$ for Cabibbo-suppressed decay) primary process is considered and the $\bar{u} d$ pair is hadronized inserting an extra $\bar{q} q$ pair using the ${ }^{3} P_{0}$ model [19-21]. The novelty of the approach of [18] is that an elaborate angular momentum algebra calculation is performed that allows one to relate the different processes, up to a global form factor from the matrix element of the quark radial wave functions. This approach is in line with the one followed for the $B \rightarrow M_{1} M_{2}$ weak decay in [22]. As far as this form factor is similar for $\tau^{-} \rightarrow \nu_{\tau} K^{* 0} \bar{K}$ or $\tau^{-} \rightarrow \nu_{\tau} K^{* 0} \bar{K}^{*}$ the ratio of the decay rates can be calculated, and one can also relate cases like $\tau^{-} \rightarrow \nu_{\tau} K^{* 0} K^{-}$with $\tau^{-} \rightarrow \nu_{\tau} \rho^{0} K^{-}, \tau^{-} \rightarrow \nu_{\tau} \omega K^{-}, \tau^{-} \rightarrow \nu_{\tau} K^{*-} \eta$ etc. Except in cases with $\pi$ production where the form factors are rather different than for other processes, the results obtained are in fair agreement with experiments.

Another good feature of this approach is that it provides directly the contribution for each value of the vector spin polarization. In [18] and $[6,15-17]$ the sum over polarizations of $\tau, \nu_{\tau}$ and the vector is performed. The purpose of the present work is to evaluate explicitly the contribution of each polarizations component, and we shall see that they are rather different, and in view of this, we perform the calculations for the weak operator of the hadronic vertex $\gamma^{\mu}-\alpha \gamma^{\mu} \gamma_{5}[\alpha=1$ for the Standard Model (SM)] that one encounters in models 
beyond the Standard Model (BSM), and conclude that the experimental investigation of these magnitudes can bring valuable information concerning extrapolations of the Standard Model. Although this type of Lagrangian does not include the different extrapolations BSM [23-25, 27-29, 31, 56, 57]. (see also the mini-review "Muon decay parameters" in PDG [32]), the results obtained for this sub-set of BSM models is rather illustrative, as we shall see.

Interestingly, such studies have been conducted in related reactions, as the semileptonic decay of mesons, like the $B \rightarrow D^{*} \bar{\nu} l$. Indeed, helicity amplitudes are evaluated in [33] and longitudinal and transverse polarizations are also separated in the $B \rightarrow D^{*} \bar{\nu}_{\tau} \tau$ decay in [34]. In [35] we performed also the calculations of the $D^{*}$ helicity amplitudes for the $B \rightarrow D^{*} \bar{\nu} l$ decay and extrapolated them for the general case of $\gamma^{\mu}-\alpha \gamma^{\mu} \gamma_{5}$ weak quark vertex, varying the value of $\alpha$ to accommodate potential models BSM. We found that the results, in particular the difference of contributions from $M=-1$ and $M=+1$, were very sensitive to $\alpha$, rendering this magnitude a very good instrument to advance in our comprehension of the Standard Model and beyond.

The present work follows the idea of [35] and also evaluates the contributions of $M=$ $0, \pm 1$, the third component of the $K^{* 0}$ spin along the direction of the $\nu_{\tau}$, for the $\tau^{-} \rightarrow$ $\nu_{\tau} K^{* 0} K^{-}$reactions. The approach of [18] is most suited to this study since what is missing in this approach is a form factor coming from the integral of $j_{0}(q r)$ with the radial part of the linear quark wave functions involved, with $q$ the momentum transfer. Yet, this form factor cancels exactly in the ratios of amplitudes, which in our approach are then produced without any free parameter. As in [35] here we also find a big sensitivity of the ratios of the polarization amplitudes to the value of $\alpha$ in $\gamma^{\mu}-\alpha \gamma^{\mu} \gamma_{5}$, which converts the study of these magnitudes into an excellent tool to further learn about the weak interaction and the possible influence of new physics.

\section{FORMALISM}

We will study the polarization amplitudes for $\tau^{-} \rightarrow \nu_{\tau} K^{* 0} K^{-}$. As shown in [18], the reaction proceeds in $s$-wave for $K^{* 0} K^{-}$and the results correspond to the case $J=1, J^{\prime}=0$ studied there with $J$ the spin of the $K^{* 0}$ and $J^{\prime}$ the one of $K^{-}$. Diagrammatically the reaction proceeds as shown in Fig. 1. The $W$ produces a $d \bar{u}$ quark pair $(s \bar{u}$ in the Cabibbo suppressed case) which hadronizes due to the creation of a $\bar{q} q$ pair with the quantum numbers 


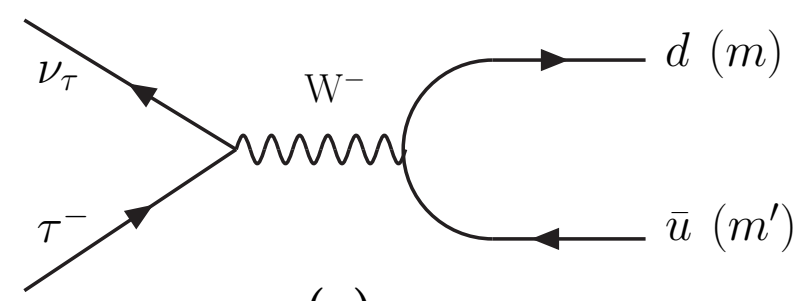

(a)

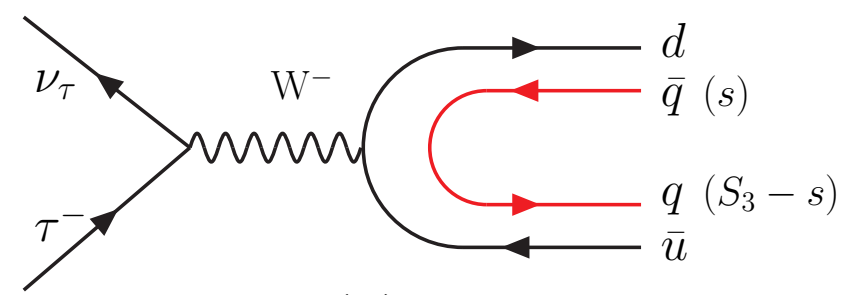

(b)

FIG. 1. (a) Elementary $\tau^{-} \rightarrow \nu_{\tau} d \bar{u}$ diagram. The labels $m, m^{\prime}$ stand for the third component of spin of the quarks; (b) Hadronization of the primary $d \bar{u}$ pair to produce two mesons, $s$ is the third component of the spin of $\bar{q}$ propagating as a particle, while $S_{3}-s$ is the third component of the spin of $q$, where $S_{3}$ is the third component of the total spin $S$ of $\bar{q} q$.

of the vacuum. The hadronization is taken into account by means of the ${ }^{3} P_{0}$ model, and by taking $\bar{q} q=\bar{u} u+\bar{d} d+\bar{s} s$ we can relate different flavors in the $\tau^{-} \rightarrow \nu_{\tau} V M$ [18]. However, since we only wish to investigate $\tau^{-} \rightarrow \nu_{\tau} K^{* 0} K^{-}$, it is sufficient to see that this mode is obtained hadronizing $d \bar{u}$ with $\bar{s} s$. The elementary quark interaction is given by

$$
H=\mathcal{C} L^{\mu} Q_{\mu}
$$

where the $\mathcal{C}$ contains the couplings of the weak interaction. The constant $\mathcal{C}$ plays no role in our study because we are only concerned about ratios of rates. The leptonic current is given by

$$
L^{\mu}=\left\langle\bar{u}_{\nu}\left|\gamma^{\mu}-\gamma^{\mu} \gamma_{5}\right| u_{\tau}\right\rangle
$$

and the quark current by

$$
Q^{\mu}=\left\langle\bar{u}_{d}\left|\gamma^{\mu}-\gamma^{\mu} \gamma_{5}\right| v_{\bar{u}}\right\rangle
$$

As is usual in the evaluation of decay widths to three final particles, we evaluate the matrix elements in the frame where the two mesons system is at rest. For the evaluation of the 
matrix element $Q_{\mu}$ we assume that the quarks are at rest in that frame and we have in the Itzykson-Zuber normalization [36] the spinors

$$
\begin{gathered}
u_{r}=\left(\begin{array}{c}
\chi_{r} \\
0
\end{array}\right), v_{r}=\left(\begin{array}{c}
0 \\
\chi_{r}
\end{array}\right), \chi_{1}=\left(\begin{array}{l}
1 \\
0
\end{array}\right), \chi_{2}=\left(\begin{array}{l}
0 \\
1
\end{array}\right), \\
Q_{0}=\left\langle\chi^{\prime}|1| \chi\right\rangle \equiv M_{0} \\
Q_{i}=\left\langle\chi^{\prime}\left|\sigma_{i}\right| \chi\right\rangle \equiv N_{i} .
\end{gathered}
$$

Even if the mesons are at rest, the quarks will have some internal momentum. This would require to use the small components in the spinors, but they contribute as $\left(p_{i n} / 2 m\right)^{2}$, and with values of $p \simeq 200 \mathrm{MeV} / c$ and an average constituent mass of $400 \mathrm{MeV}$ the effects are of the order of $6 \%$, which are assumed in this approach. Small as these effects are, they further tend to cancel in ratios of amplitudes, such that we should not worry about them.

To obtain the $\tau$ width, we must evaluate

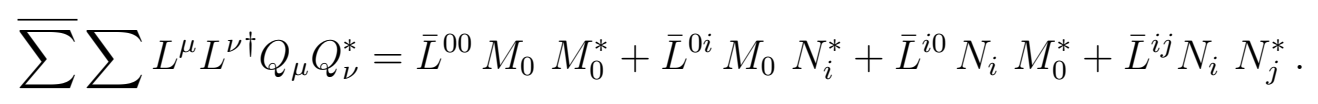

Denoting for simplicity,

$$
\begin{aligned}
\bar{L}^{\mu \nu} & =\overline{\sum \sum} L^{\mu} L^{\nu \dagger} \\
& =\frac{1}{m_{\nu} m_{\tau}}\left(p^{\prime \mu} p^{\nu}+p^{\prime \nu} p^{\mu}-g^{\mu \nu} p^{\prime} \cdot p+i \epsilon^{\alpha \mu \beta \nu} p_{\alpha}^{\prime} p_{\beta}\right),
\end{aligned}
$$

where $p, p^{\prime}$ are the momenta of the $\tau$ and $\nu_{\tau}$ respectively and we use the field normalization for fermions of Ref. [37].

For the $J=1, J^{\prime}=0$ (vector- pseudoscalar) case, we obtain the results [18]:

$$
\begin{gathered}
M_{0}=\frac{1}{\sqrt{6}} \frac{1}{4 \pi} \delta_{M^{\prime} 0} \\
N_{\mu}=(-1)^{-\mu} \frac{1}{\sqrt{3}} \frac{1}{4 \pi} \mathcal{C}(111 ; M,-\mu, M-\mu) \delta_{M^{\prime} 0}
\end{gathered}
$$

with $\mathcal{C}(\cdots)$ a Clebsch-Gordan coefficient. The $\mu$ index of Eq. (9) is the index of $N_{i}$ in spherical basis

$$
N_{+1}=-\frac{1}{\sqrt{2}}\left(N_{1}+i N_{2}\right), \quad N_{-1}=\frac{1}{\sqrt{2}}\left(N_{1}-i N_{2}\right), \quad N_{0}=N_{3} .
$$

This formalism is demanded when one has to project over spin components. In addition, $M, M^{\prime}$ are the third components of the $K^{* 0}$ and $K^{-}$respectively (obviously $M^{\prime}=0$, do 
not confuse $M, M^{\prime}$ with the amplitude $M_{0}$ of Eqs.(5),(6) and (8)). The quantization axis is taken along the direction of the neutrino in the $\tau^{-}$rest frame.

Using Eqs. (6),(7) and (8),(9) and following the steps of the appendix A, we obtain the $\tau$ decay amplitude, $t$, up to a global constant, and we find

1) $M=0$

$$
\bar{\sum} \sum|t|^{2}=\frac{1}{m_{\tau} m_{\nu}} \frac{1}{6}\left(\frac{1}{4 \pi}\right)^{2}\left(3 E_{\tau} E_{\nu}-p^{2}\right)
$$

2) $M=1$

$$
\bar{\sum} \sum|t|^{2}=\frac{1}{m_{\tau} m_{\nu}} \frac{1}{6}\left(\frac{1}{4 \pi}\right)^{2}\left[3 E_{\tau} E_{\nu}+p^{2}+\left(3 E_{\nu}+E_{\tau}\right) p\right]
$$

3) $M=-1$

$$
\bar{\sum} \sum|t|^{2}=\frac{1}{m_{\tau} m_{\nu}} \frac{1}{6}\left(\frac{1}{4 \pi}\right)^{2}\left[3 E_{\tau} E_{\nu}+p^{2}-\left(3 E_{\nu}+E_{\tau}\right) p\right]
$$

where $p$ is the momentum of the $\tau$, or $\nu_{\tau}$, in the $K^{* 0} K^{-}$rest frame, given by

$$
p=p_{\nu}=p_{\tau}=\frac{\lambda^{1 / 2}\left(m_{\tau}^{2}, m_{\nu}^{2}, M_{\mathrm{inv}}^{2\left(K^{* 0} K^{-}\right)}\right)}{2 M_{\mathrm{inv}}^{\left(K^{* 0} K^{-}\right)}},
$$

$E_{\tau}=\sqrt{m_{\tau}^{2}+p^{2}}, E_{\nu}=p$ and $\bar{L}^{\mu \nu}$ of Eq. (7) is evaluated in this frame too.

\section{RESULTS}

In the former equations the angle integrations are already done in a way that finally we

must take into account the full phase space with the angle independent expressions. So, we have

$$
\frac{d \Gamma}{d M_{\mathrm{inv}}^{\left(K^{* 0} K^{-}\right)}}=\frac{2 m_{\tau} 2 m_{\nu}}{(2 \pi)^{3}} \frac{1}{4 m_{\tau}^{2}} p_{\nu} \widetilde{p}_{1} \bar{\sum} \sum|t|^{2}
$$

where $p_{\nu}$ is the neutrino momentum in the $\tau$ rest frame

$$
p_{\nu}=\frac{\lambda^{1 / 2}\left(m_{\tau}^{2}, m_{\nu}^{2}, M_{\mathrm{inv}}^{2\left(K^{* 0} K^{-}\right)}\right)}{2 m_{\tau}}
$$

and $\widetilde{p}_{1}$ the momentum of $K^{* 0}$ in the $K^{* 0} K^{-}$rest frame given by

$$
\widetilde{p}_{1}=\frac{\lambda^{1 / 2}\left(M_{\mathrm{inv}}^{2\left(K^{* 0} K^{-}\right)}, m_{K^{* 0}}^{2}, m_{K^{-}}^{2}\right)}{2 M_{\mathrm{inv}}^{\left(K^{* 0} K^{-}\right)}} .
$$


The total differential width is given by

$$
R=\left.\frac{d \Gamma}{d M_{\mathrm{inv}}^{\left(K^{* 0} K^{-}\right)}}\right|_{M=+1}+\left.\frac{d \Gamma}{d M_{\mathrm{inv}}^{\left(K^{* 0} K^{-}\right)}}\right|_{M=0}+\left.\frac{d \Gamma}{d M_{\mathrm{inv}}^{\left(K^{* 0} K^{-}\right)}}\right|_{M=-1} .
$$

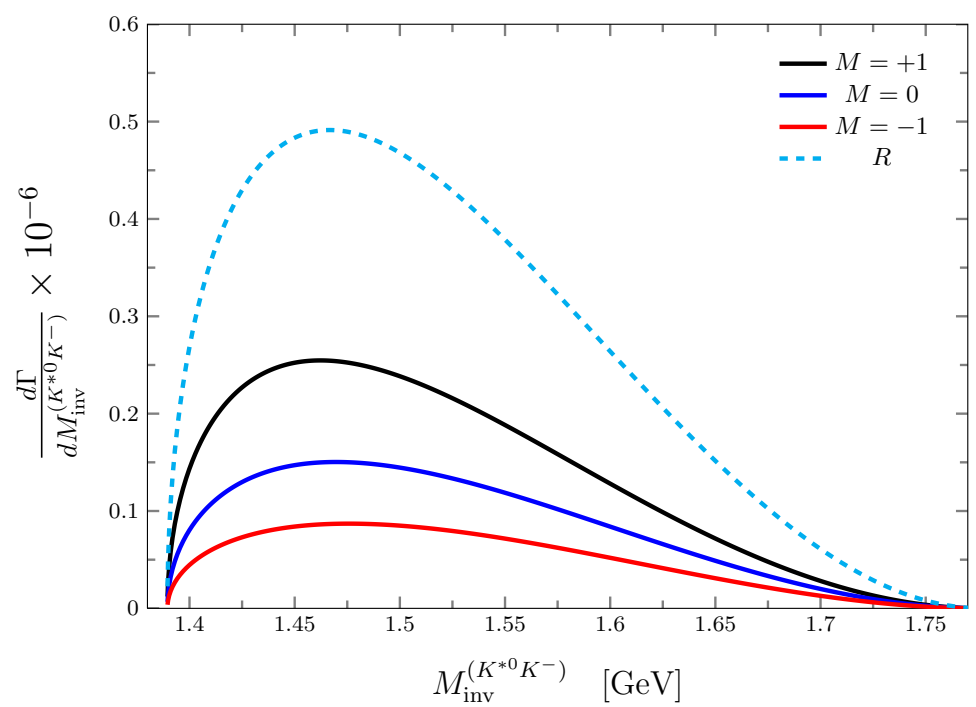

FIG. 2. Total differential width $R$ of Eq. (18), and individual contributions of $\left.\frac{d \Gamma}{d M_{\mathrm{inv}}^{\left(K^{-} K^{* 0}\right)}}\right|_{M=0}$, $\left.\frac{d \Gamma}{d M_{\text {inv }}^{\left(K^{-} K^{* 0}\right)}}\right|_{M=-1}$, and $\left.\frac{d \Gamma}{d M_{\text {inv }}^{\left(K^{-} K^{* 0}\right)}}\right|_{M=+1}$.

In Fig. 2 we show the individual contribution of each $M$ and the total $R$. In Fig. 3 we show the contribution of each $M$ and the difference of $M=+1$ and $M=-1$, divided by the total differential width $R$.

In the search for contributions BSM one usually compares some magnitude with experiment and diversions of experiment with respect to the SM predictions are seen as a signal of possible new physics. So far the experimental errors do not make the cases compelling. The present case could offer a good opportunity, since the individual contributions for different $M$ vary appreciably when diverting from the Standard Model, as we show in the next section. 


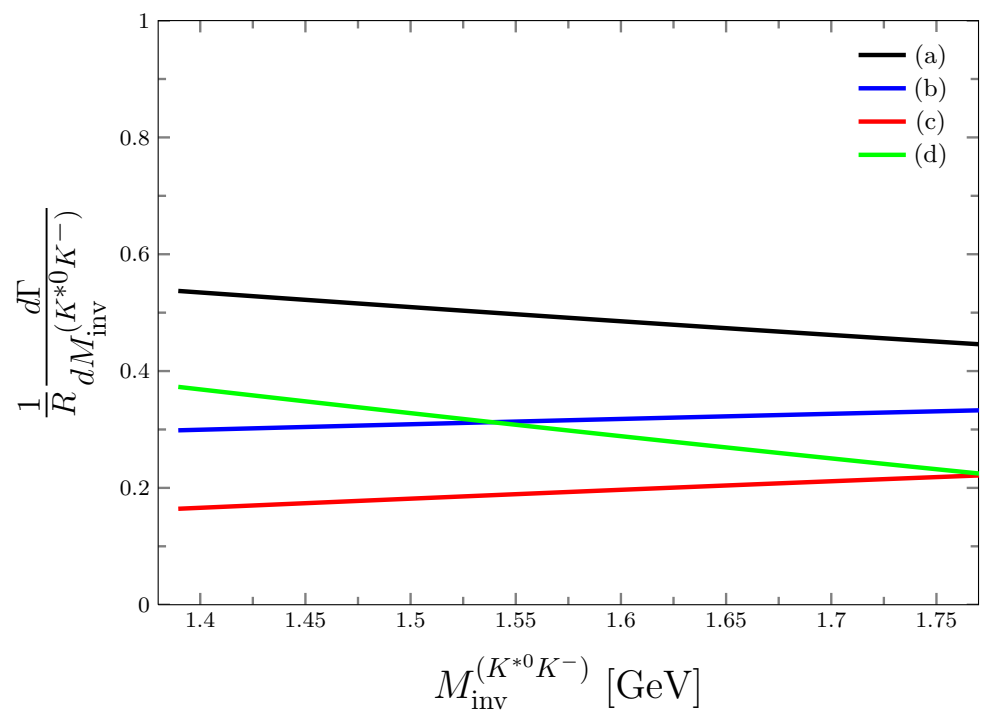

FIG. 3. The different ratios, where lines (a), (b) and (c) show $\left.\frac{d \Gamma}{d M_{\text {inv }}^{\left(K^{-} K^{* 0)}\right.}}\right|_{M=+1},\left.\frac{d \Gamma}{d M_{\text {inv }}^{\left(K^{-} K^{* 0}\right)}}\right|_{M=0}$, and $\left.\frac{d \Gamma}{d M_{\mathrm{inv}}^{\left(K^{-} K^{* 0}\right)}}\right|_{M=-1}$ respectively, and line (d) denotes the difference of $\left.\frac{d \Gamma}{d M_{\mathrm{inv}}^{\left(K^{-} K^{* 0}\right)}}\right|_{M=+1}-$ $\left.\frac{d \Gamma}{d M_{\mathrm{inv}}^{\left(K^{-} K^{* 0}\right)}}\right|_{M=-1}$, all divided by the total differential width $R$ of Eq. (18).

\section{CONSIDERATION OF RIGHT-HANDED QUARK CURRENTS}

There is a huge amount of work on extensions of the Standard Model and this is not the place to discuss it. We only like to mention current models which are widely used recently, as minimal gauge extensions of the SM [23, 24], leptoquarks[25], scalar leptoquarks [27, 56], Pati-Salam gauge models [28, 29, 57] and right-handed models [31, 38].

Some models BSM have quark currents that contain the combination $\gamma^{\mu}+\gamma^{\mu} \gamma_{5}$. Some of the models mentioned above could be accounted for with an operator

$$
\begin{aligned}
& a\left(\gamma^{\mu}-\gamma^{\mu} \gamma_{5}\right)+b\left(\gamma^{\mu}+\gamma^{\mu} \gamma_{5}\right) \\
= & (a+b)\left\{\gamma^{\mu}-\frac{a-b}{a+b} \gamma^{\mu} \gamma_{5}\right\} .
\end{aligned}
$$

We shall call $\frac{a-b}{a+b}=\alpha$ and study the distributions for different $M$ as a function of $\alpha$. Thus, we have the operator

$$
\gamma^{\mu}-\alpha \gamma^{\mu} \gamma_{5}
$$

Using the same formalism of [18], and the appendix A of the present work, it is easy to see the results as a function of $\alpha$. We obtain the following results: 

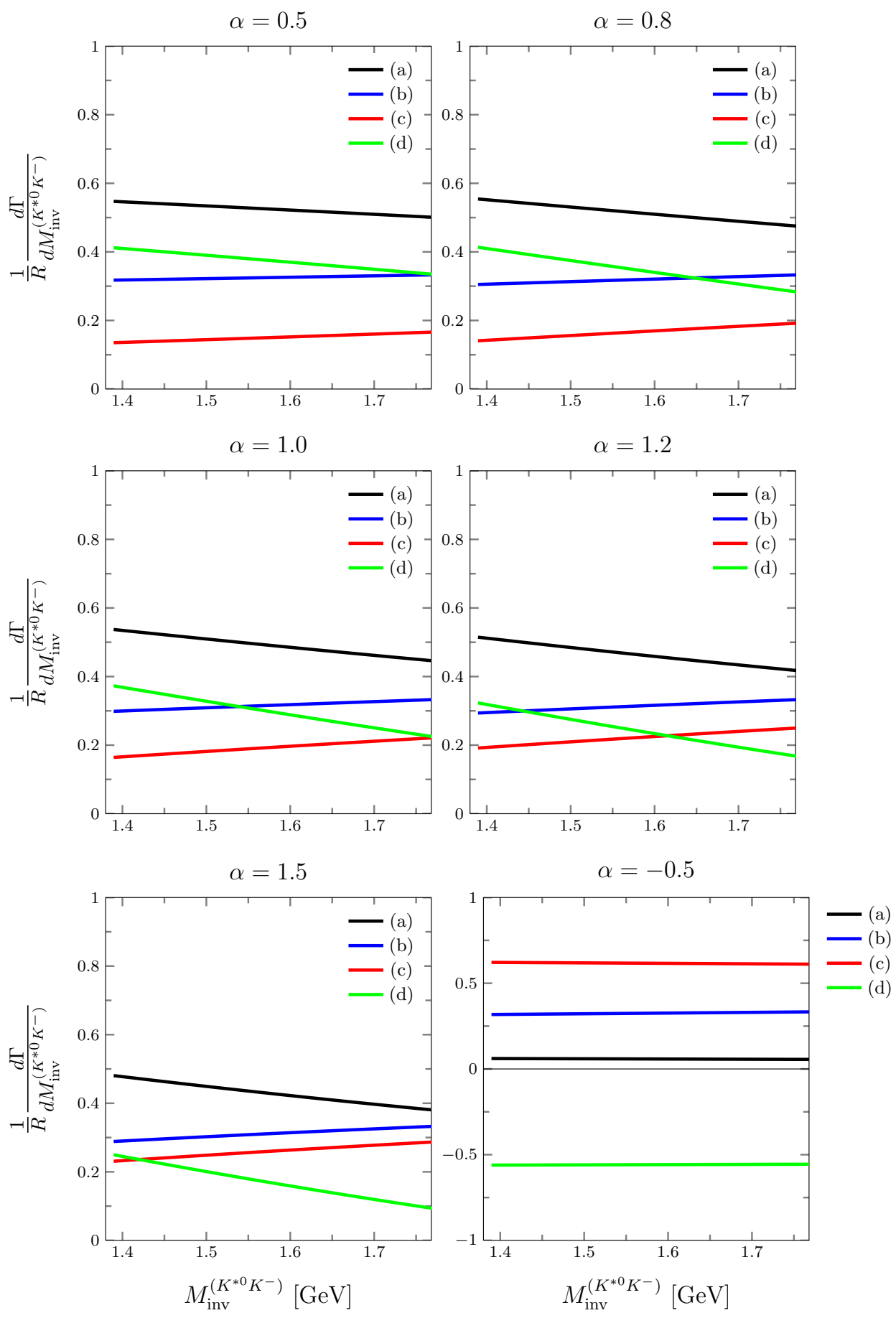

FIG. 4. The same as Fig. 3 but for different $\alpha$.

1) $M=0$

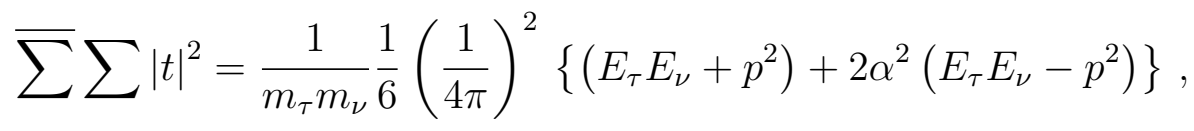


2) $M=1$

$$
\begin{aligned}
\overline{\sum \sum|t|^{2}} & =\frac{1}{m_{\tau} m_{\nu}} \frac{1}{6}\left(\frac{1}{4 \pi}\right)^{2}\left\{\left(E_{\tau} E_{\nu}+p^{2}\right)+2 \alpha\left(E_{\nu}+E_{\tau}\right) p\right. \\
& \left.+\left[2 E_{\tau} E_{\nu}+\left(E_{\nu}-E_{\tau}\right) p\right] \alpha^{2}\right\}
\end{aligned}
$$

3) $M=-1$

$$
\begin{aligned}
\overline{\sum \sum|t|^{2}} & =\frac{1}{m_{\tau} m_{\nu}} \frac{1}{6}\left(\frac{1}{4 \pi}\right)^{2}\left\{\left(E_{\tau} E_{\nu}+p^{2}\right)-2 \alpha\left(E_{\nu}+E_{\tau}\right) p\right. \\
& \left.+\left[2 E_{\tau} E_{\nu}-\left(E_{\nu}-E_{\tau}\right) p\right] \alpha^{2}\right\}
\end{aligned}
$$

In Fig. 4 we show the results for values of $\alpha$ not too different from unity. As we can see, the results of the polarization amplitudes vary very much with $\alpha$. These are large changes that could not scape a precise experimental determination of these magnitudes. In particular we find a magnitude which is extremely sensitive to $\alpha$ which is $\left.\frac{1}{R} \frac{d \Gamma}{d M_{\text {inv }}^{\left(K^{* 0} K^{-}\right)}}\right|_{M=+1}-\left.\frac{1}{R} \frac{d \Gamma}{d M_{\text {inv }}^{\left(K^{* 0} K^{-}\right)}}\right|_{M=-1}$. We show this magnitude in Fig. 5 for different values of $\alpha$. This magnitude changes even sign for some value of $\alpha$ and turns out to be most suited to investigate possible deviations of the SM.

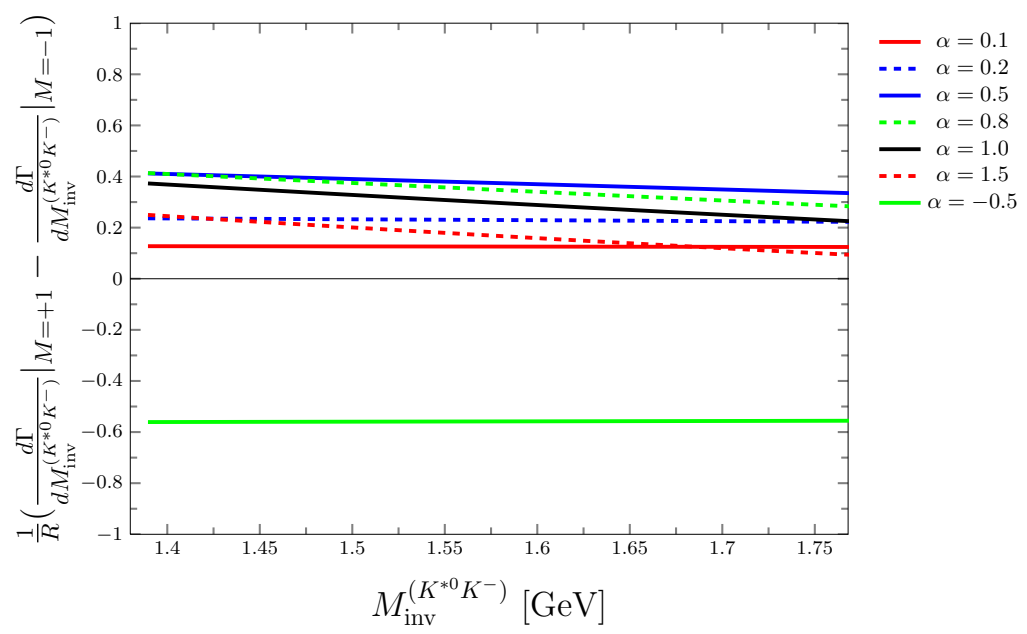

FIG. 5. The difference of $\left.\frac{d \Gamma}{d M_{\mathrm{inv}}^{\left(K^{* 0} K^{-}\right)}}\right|_{M=+1}-\left.\frac{d \Gamma}{d M_{\mathrm{inv}}^{\left(K^{* 0} K^{-}\right)}}\right|_{M=-1}$ as a function of $\alpha$, divided by the total differential width $R$. 


\section{FINAL STATE INTERACTION AND RESONANCE PRODUCTION, CON- NECTION WITH OTHER FORMALISMS}

We have used a contact interaction for the evaluation of the $\tau^{-} \rightarrow \nu_{\tau} K^{* 0} K^{-}$amplitudes. However, it is well known that axial vector resonance, like the $a_{1}(1260)$ have contribution to this process, as evidenced in the related $\pi^{-} \rho$ channel $[7,39,40]$. From our perspective, the contribution of these resonances appears considering the final state interaction of the components that come from the hadronization of $d \bar{u}$. As shown in [18], the combination stemming from this hadronization is given by

$$
(V \cdot P)_{21}=\rho^{-}\left(\frac{\pi^{0}}{\sqrt{2}}+\frac{\eta}{\sqrt{3}}+\frac{\eta^{\prime}}{\sqrt{6}}\right)+\left(-\frac{\rho^{0}}{\sqrt{2}}+\frac{\omega}{\sqrt{2}}\right) \pi^{-}+K^{* 0} K^{-} .
$$

This means that the $K^{* 0} K^{-}$in the final state is reached by the series of diagrams depicted in Fig. 6, where $V_{i} P_{i}$ is any of the coupled channels that appears in Eq. (23). This means

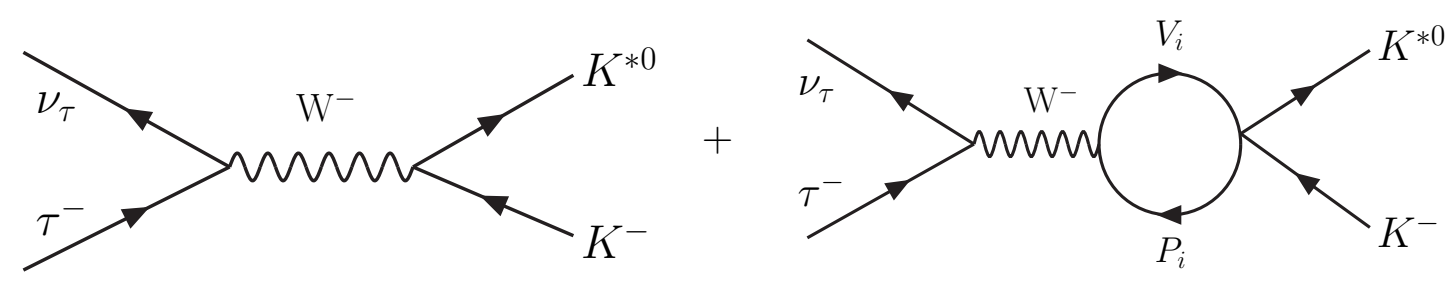

FIG. 6. Diagrammatic representation of $K^{* 0} K^{-}$production with final state interaction of coupled $V_{i} P_{i}$ channels.

that we must multiply the elementary amplitude previously evaluated by

$$
1+\sum_{i} G_{i} t_{i, K^{* 0} K^{-}}
$$

to obtain the final amplitude, where $G_{i}$ is the vector-pseudoscalar loop function and $t_{i, K^{* 0} K^{-}}$ the transition amplitude from channel $i$ to $K^{* 0} K^{-}$. These magnitudes are evaluated in [4143] using the chiral unitary approach for the $V P$ interaction in $s$-wave. The important thing for the present work is that the $t_{i j}$ matrix conserves the spin of the vector, with the vertex $\boldsymbol{\epsilon} \cdot \boldsymbol{\epsilon}^{\prime}$, and hence, the polarization in all the vectors in Fig. 6 is the same, and the interaction factor of Eq. (24) is independent of spin. As a consequence, while all the polarization amplitudes will be affected by this interaction, that generates dynamically the axial vector resonances, the different polarization amplitudes are affected by the same factor and in the ratios it cancels exactly. 
Next we would like to make connection to the conventional representation of the amplitudes in terms of invariants that contain the polarization vector of the $K^{*}$. For this purpose we find useful to use the formalism of $[44,45]$ in the related semileptonic decay $K^{*} \rightarrow l \bar{\nu} K$. By analogy to $B \rightarrow D^{*} \nu l$ in $[44,45]$ we write

$$
\begin{gathered}
\left\langle K^{*}, \lambda, P_{K^{*}}\left|\mathcal{J}_{\mu}(0)\right| K, P_{K}\right\rangle=\frac{2 i V\left(q^{2}\right)}{m_{K}+m_{K^{*}}} \epsilon_{\mu \nu \alpha \beta}\left(\epsilon^{(\lambda) \nu}\right)^{*} P_{K}^{\alpha} P_{K^{*}}^{\beta}-2 m_{K^{*}} A_{0}\left(q^{2}\right) \frac{\epsilon^{(\lambda) *} \cdot q}{q^{2}} q_{\mu} \\
-\left(m_{K}+m_{K^{*}}\right) A_{1}\left(q^{2}\right)\left[\epsilon_{\mu}^{(\lambda) *}-\frac{\epsilon^{(\lambda) *} \cdot q}{q^{2}} q_{\mu}\right] \\
+A_{2}\left(q^{2}\right) \frac{\epsilon^{(\lambda) *} \cdot q}{m_{K}+m_{K^{*}}}\left[\left(P_{K}+P_{K^{*}}\right)_{\mu}-\frac{m_{K}^{2}-m_{K^{*}}^{2}}{q^{2}} q_{\mu}\right]
\end{gathered}
$$

where $q=P_{K}-P_{K^{*}}$ (in the present case since $K^{*}, K$ are both produced $q=P_{K}+P_{K^{*}}=$ $\left.P_{\tau}-P_{\nu}\right)$. In [35] the connection of this formalism to our formalism in terms of the explicit $M=0, \pm 1$ amplitudes is done. In the present case it is unnecessary to repeat the procedure because we can take advantage of the results found on [35] to justify our results. Indeed, it might appear surprising that the formalism of Eq. (25) requires four form factors while we could make predictions with no free parameters. For this we can go to Ref. [35] (Fig. 5 and Fig. 10) where one observes that the behaviour of the different $M$ contributions to $\frac{d \Gamma}{d M_{\mathrm{inv}}^{(\nu l)}}$ at $M_{\mathrm{inv}}^{(\nu l)} / \max$, divided by the total differential width $R$ of Eq. (18), is exactly the same in our approach as for the Standard Model using the empirical values of the four form factors. The reason was found in [35] because at $M_{\mathrm{inv}}^{(\nu l)}$ maximum only the $A_{1}\left(q^{2}\right)$ amplitude contributes. In addition it was found that this term dominates in a region of $M_{\mathrm{inv}}^{(\nu l)}$ values close the the maximum (in a range of $500 \mathrm{MeV}$ where our results are practically indistinguishable from those of the Standard Model). As a consequence of that, our approach providing a slightly different functional of $A_{1}\left(q^{2}\right)$ than the Standard Model gave basically the same results of the Standard Model in the ratios of amplitudes, where $A_{1}\left(q^{2}\right)$ cancels. In the present case there is not much phase space in the $\tau^{-} \rightarrow \nu_{\tau} K^{* 0} K^{-}$reaction, and the distance of the peak

of the invariant mass distribution to $M_{\mathrm{inv}}^{(\nu l)} / \max$ is about $250 \mathrm{MeV}$ [7], so it falls well within the range of dominance of $A_{1}\left(q^{2}\right)$, which guarantees that it will cancel in ratios, which can then be obtained with no parameters as it has naturally appeared in our approach.

\section{FINAL REMARKS}

The $\tau$ decays have been the subject of intense research, both experimental and theoretical. Ongoing discussion continues and there are prospects for construction of new facilities, one 
of them at Hefei [46], another one at Novosibirsk [47] (see also " workshop on future taucharm factory" [48]). In addition, the new Belle upgrade will devote further attentions to the subject [49].

Polarization measurements have been addressed in related semileptonic reactions and can be well adapted to $\tau$ decays. The separation of the polarization amplitudes can be accomplished by looking at the decay products of $K^{*} \rightarrow K \pi$ and angular correlations between the momenta of these particles and the momentum of the $\tau, \nu$ in the rest frame of $K^{*} \bar{K}$ $[34,50-52]$.

Finally we should also remark that the form of quark operator to go beyond the Standard Model that we have adopted [Eq. (19)] does not account for all the large variety of models BSM. It would be closet to the Right-Left handed models used in [31, 53-57]. However, the fact that we have seen much sensitivity of the polarization results in this subset of BSM models, should be an incentive for the study of these observables within other models, such that future experiments can serve to elucidate between them.

\section{CONCLUSIONS}

We have performed a calculation of the different vector meson polarization contribution for a $\tau^{-} \rightarrow \nu_{\tau} V M$ decay, concretly $\tau^{-} \rightarrow \nu_{\tau} K^{* 0} K^{-}$. The $M= \pm 1,0$ third components of the $K^{* 0}$ spin are taken with respect to a frame where the $z$ axis is chosen in the neutrino

direction. In a first step we evaluate these contributions to $\frac{d \Gamma}{d M_{\mathrm{inv}}^{\left(K^{* 0} K^{-}\right)}}$within the Standard Model and we see that they differ from each other appreciably. In view of that we extend the calculations to the case of one weak hadronic vertex of the type $\gamma^{\mu}-\alpha \gamma^{\mu} \gamma_{5}$, which when $\alpha \neq 1$ can accommodate a sub-set of models beyond the Standard Model. We find that the results depend strongly on $\alpha$ and particularly the magnitude $\left.\frac{d \Gamma}{d M_{\text {inv }}^{\left(K^{* 0} K^{-}\right)}}\right|_{M=+1}-\left.\frac{d \Gamma}{d M_{\text {inv }}^{\left(K^{* 0} K^{-}\right)}}\right|_{M=-1}$ divided by the sum of the three contributions $(M= \pm 1,0)$, which changes even sign for some values of $\alpha$. We also discussed that resonance production in these reactions, mostly through excitation of axial vector resonances, modifies the contribution of the different $M$ amplitudes but not their ratio. Furthermore, by making a comparison with the invariant amplitude formalism with explicit polarization vectors, by analogy to studies of the semileptonic decay of hadrons which involves four invariant form factors, we found that the present amplitudes are dominated by just the $A_{1}\left(q^{2}\right)$ form factor, which then cancels in ratios, such that they 
can be calculated with no free parameters as we have done in our approach. In view of all this, we propose the measurement of these polarization magnitudes, which are bound to provide new light on the Standard Model and possible extensions beyond the Standard Model.

\section{ACKNOWLEDGMENTS}

We wish to express our thanks to Jose Valle, Martin Hirsch, Avelino Vicente, XiaoGang He, Juan Nieves and Eliecer Hernandez for useful discussions. LRD acknowledges the support from the National Natural Science Foundation of China (Grant No. 11575076) and the State Scholarship Fund of China (No. 201708210057). This work is partly supported by the Spanish Ministerio de Economia y Competitividad and European FEDER funds under Contracts No. FIS2017-84038-C2-1-P B and No. FIS2017-84038-C2-2-P B, and the Generalitat Valenciana in the program Prometeo II-2014/068, and the project Severo Ochoa of IFIC, SEV-2014-0398 (EO).

\section{Appendix A: Evaluation of $\bar{\sum} \sum|t|^{2}$}

We start from Eqs. (6),(7) and follow the nomenclature $\bar{L}^{\mu \nu}=\bar{\sum} \sum L^{\mu} L^{\dagger \nu}$ adopted before for simplicity, we have for the leptonic sector

$$
\begin{aligned}
\bar{L}^{\mu \nu} \equiv \overline{\sum \sum L^{\mu} L^{\dagger \nu}} & =\frac{1}{m_{\tau} m_{\nu}}\left\{p^{\prime \mu} p^{\nu}+p^{\prime \nu} p^{\mu}-g^{\mu \nu}\left(p^{\prime} \cdot p\right)\right. \\
& \left.+i \epsilon^{\alpha \mu \beta \nu} p_{\alpha}^{\prime} p_{\beta}\right\}
\end{aligned}
$$

Recall that we take the direction of the neutrino as the quantum $z$ axis and evaluate the different magnitudes in the $K^{* 0} K^{-}$rest frame.

Thus, for the leptonic plus hadronic matrix elements we have

$$
\bar{\sum} \sum|t|^{2}=\bar{L}^{00} M_{0} M_{0}^{*}+\bar{L}^{0 i} M_{0} N_{i}^{*}+\bar{L}^{i 0} N_{i} M_{0}^{*}+\bar{L}^{i j} N_{i} N_{j}^{*}
$$


1. For $M=0$

1) $\bar{L}^{00} M_{0} M_{0}^{*}$ term

$$
\begin{aligned}
\bar{L}^{00} & =\frac{1}{m_{\tau} m_{\nu}}\left[E_{\nu} E_{\tau}+E_{\tau} E_{\nu}-\left(p_{\nu} \cdot p_{\tau}\right)\right] \\
& =\frac{1}{m_{\tau} m_{\nu}}\left(2 E_{\nu} E_{\tau}-E_{\tau} E_{\nu}+\boldsymbol{p}_{\tau} \cdot \boldsymbol{p}_{\nu}\right) \\
& =\frac{1}{m_{\tau} m_{\nu}}\left(E_{\nu} E_{\tau}+\boldsymbol{p}_{\nu}^{2}\right)
\end{aligned}
$$

Recall that in that frame of reference $\boldsymbol{p}_{\tau}=\boldsymbol{p}_{\nu}=\boldsymbol{p}$. Using Eq. (8), we obtain

$$
\bar{L}^{00} M_{0} M_{0}^{*}=\frac{1}{m_{\tau} m_{\nu}}\left(\frac{1}{\sqrt{6}} \frac{1}{4 \pi}\right)^{2}\left(E_{\tau} E_{\nu}+p^{2}\right)
$$

2) $\bar{L}^{0 i} M_{0} N_{i}^{*}$ and $\bar{L}^{i 0} N_{i} M_{0}^{*}$ terms

$$
\bar{L}^{0 i}=\frac{1}{m_{\tau} m_{\nu}}\left[p_{\nu}^{0} p_{\tau}^{i}+p_{\tau}^{0} p_{\nu}^{i}-g^{0 i}\left(p_{\nu}^{0} \cdot p_{\tau}^{i}\right)+i \epsilon^{\alpha 0 \beta i} p_{\nu \alpha} p_{\tau \beta}\right]
$$

where $g^{0 i}=0$, and for $i \epsilon^{\alpha 0 \beta \nu}, \alpha, \beta$ are spatial, $\boldsymbol{p}_{\tau}=\boldsymbol{p}_{\nu}$, thus $\epsilon^{\alpha 0 \beta \nu} p_{\nu \alpha} p_{\tau \beta}=0$.

Now let us use the relationship

$$
\sum_{i} p_{\tau}^{i} N_{i}^{*}=\sum_{\mu} p_{\tau \mu} N_{\mu}^{*}
$$

with $\mu$ in spherical basis, and since we take $\boldsymbol{p}$ in the $z$ direction,

$$
\sum_{i} p_{\tau}^{i} N_{i}^{*}=p_{\tau z} N_{0}^{*}
$$

and using Eq.(9),

$$
N_{0}=\frac{1}{\sqrt{3}} \frac{1}{4 \pi} \mathcal{C}(111 ; M, 0, M)=\frac{1}{\sqrt{3}} \frac{1}{4 \pi} \mathcal{C}(111 ; 0,0,0)=0,
$$

hence, the $\bar{L}^{0 i} M_{0} N_{i}^{*}$ term gives no contribution, and the same thing happens for the $\bar{L}^{i 0} N_{i} M_{0}^{*}$ term.

3) The $\bar{L}^{i j} N_{i} N_{j}^{*}$ term. We have

$$
\bar{L}^{i j} N_{i} N_{j}^{*}=\sum_{\alpha, \beta}(-1)^{\alpha} \bar{L}^{\alpha \beta} N_{-\alpha} N_{\beta}^{*}
$$

with $\alpha, \beta$ in spherical basis. So for different terms, we obtain 
(a) since $\boldsymbol{p}_{\tau}, \boldsymbol{p}_{\nu}$ are in the $z$ direction, we have

$$
\begin{gathered}
(-1)^{\alpha}\left(p_{\nu \alpha} p_{\tau \beta}+p_{\nu \beta} p_{\tau \alpha}\right) N_{-\alpha} N_{\beta}^{*}=\left(p^{2}+p^{2}\right) N_{0} N_{0}^{*} \\
=2 p^{2} \mathcal{C}(111 ; 0,0,0) \mathcal{C}(111 ; 0,0,0)=0 .
\end{gathered}
$$

(b) for the $-g_{i j}\left(p_{\nu} \cdot p_{\tau}\right) N_{i} N_{j}^{*}$ term, we have

$$
\begin{gathered}
-g_{i j}\left(p_{\nu} \cdot p_{\tau}\right) N_{i} N_{j}^{*}=\delta_{i j}\left(p_{\nu} \cdot p_{\tau}\right) N_{i} N_{j}^{*}=\sum_{\mu}\left(p_{\nu} \cdot p_{\tau}\right) N_{\mu} N_{\mu}^{*} \\
=\sum_{\mu} \frac{1}{3}\left(\frac{1}{4 \pi}\right)^{2} \mathcal{C}^{2}(111 ; 0,-\mu,-\mu)\left(p_{\nu} \cdot p_{\tau}\right) \\
=\frac{1}{3}\left(\frac{1}{4 \pi}\right)^{2}\left(\frac{1}{2}+0+\frac{1}{2}\right)\left(p_{\nu} \cdot p_{\tau}\right)=\frac{1}{3}\left(\frac{1}{4 \pi}\right)^{2}\left(p_{\nu} \cdot p_{\tau}\right) .
\end{gathered}
$$

(c) for the $\epsilon^{\alpha i \beta j} p_{\nu \alpha} p_{\tau \beta} N_{i} N_{j}^{*}$ term, since $\alpha$ or $\beta$ must be zero, so we take $\alpha=0$, then this term becomes $E_{\nu} \epsilon^{i \beta j} p_{\tau \beta} N_{i} N_{j}^{*}$, after taking $\beta=3$ which means $z$ direction or third component, and using Eqs. (8) and (9), we obtain

$$
\begin{aligned}
i E_{\nu} \epsilon^{i 3 j} p N_{i} N_{j}^{*}= & -i E_{\nu} \epsilon^{3 i j} p N_{i} N_{j}^{*}=-i E_{\nu} p\left(N_{1} N_{2}^{*}-N_{2} N_{1}^{*}\right)=-i i E_{\nu} p\left(N_{-} N_{-}-N_{+} N_{+}\right) \\
& =E_{\nu} p\left[\mathcal{C}^{2}(111 ; 0,+1,+1)-\mathcal{C}^{2}(111 ; 0,-1,-1)\right]=0,
\end{aligned}
$$

where we use the expressions of Eq.(10) for $N_{+}$and $N_{-}$.

Altogether, from Eqs. (A4) and (A11), we obtain

$$
\begin{aligned}
\overline{\sum \sum|t|^{2}} & =\frac{1}{m_{\tau} m_{\nu}}\left[\frac{1}{6}\left(\frac{1}{4 \pi}\right)^{2}\left(E_{\tau} E_{\nu}+\boldsymbol{p}_{\nu}^{2}\right)+\frac{1}{3}\left(\frac{1}{4 \pi}\right)^{2}\left(p_{\nu} \cdot p_{\tau}\right)\right] \\
& =\frac{1}{m_{\tau} m_{\nu}} \frac{1}{6}\left(\frac{1}{4 \pi}\right)^{2}\left[\left(E_{\tau} E_{\nu}+p^{2}\right)+2\left(E_{\tau} E_{\nu}-p^{2}\right)\right]
\end{aligned}
$$

2. for $M= \pm 1$

1) $\bar{L}^{00} M_{0} M_{0}^{*}$ terms

The same result as above (A4), because $M_{0}$ is the same for all $M$,

$$
\bar{L}^{00} M_{0} M_{0}^{*}=\frac{1}{m_{\tau} m_{\nu}}\left(\frac{1}{\sqrt{6}} \frac{1}{4 \pi}\right)^{2}\left(E_{\tau} E_{\nu}+p^{2}\right)
$$

2) $\bar{L}^{0 i} M_{0} N_{i}^{*}$ and $\bar{L}^{i 0} N_{i} M_{0}^{*}$ terms

$$
\bar{L}^{0 i} M_{0} N_{i}^{*}=\frac{1}{m_{\tau} m_{\nu}}\left(p_{\nu}^{0} p_{\tau}^{i}+p_{\tau}^{0} p_{\nu}^{i}\right) M_{0} N_{i}^{*}=\frac{1}{m_{\tau} m_{\nu}}\left\{\left(E_{\tau}+E_{\nu}\right) \sum_{\mu} p_{\mu} N_{\mu}^{*}\right\} M_{0},
$$


By taking the third $p_{3}$ component ( $z$ direction in spherical basis), Eq. (A15) becomes

$$
\begin{aligned}
\bar{L}^{0 i} M_{0} N_{i}^{*} & =\frac{1}{m_{\tau} m_{\nu}}\left\{\left(E_{\tau}+E_{\nu}\right) p \frac{1}{\sqrt{6}} \frac{1}{4 \pi} \frac{1}{\sqrt{3}} \frac{1}{4 \pi}(-1)^{0} \mathcal{C}(111 ; M, 0, M)\right\} \\
& =\frac{1}{m_{\tau} m_{\nu}}\left\{\left(E_{\tau}+E_{\nu}\right) \frac{1}{6}\left(\frac{1}{4 \pi}\right)^{2} p M\right\}
\end{aligned}
$$

and $\bar{L}^{i 0} N_{i} M_{0}^{*}$ is the complex conjugate of the former one of Eq. (A16).

Altogether, we obtain

$$
\bar{L}^{0 i} M_{0} N_{i}^{*}+\bar{L}^{i 0} N_{i} M_{0}^{*}=\frac{1}{m_{\tau} m_{\nu}} \frac{1}{3}\left(\frac{1}{4 \pi}\right)^{2}\left(E_{\tau}+E_{\nu}\right) p M .
$$

3) The $\bar{L}^{i j} N_{i} N_{j}^{*}$ term

(a) since $\boldsymbol{p}_{\tau}, \boldsymbol{p}_{\nu}$ are in the $z$ direction, in analogy to Eq. (A10), but having here $M= \pm 1$, we have

$$
\begin{gathered}
\frac{1}{m_{\tau} m_{\nu}}(-1)^{\alpha}\left(p_{\nu \alpha} p_{\tau \beta}+p_{\nu \beta} p_{\tau \alpha}\right) N_{-\alpha} N_{\beta}^{*} \\
=\frac{1}{m_{\tau} m_{\nu}}\left(p^{2}+p^{2}\right) N_{0} N_{0}^{*} \\
=\frac{1}{m_{\tau} m_{\nu}} 2 p^{2} \frac{1}{3}\left(\frac{1}{4 \pi}\right)^{2} \mathcal{C}^{2}(111 ; M, 0, M)=\frac{1}{m_{\tau} m_{\nu}} \frac{1}{3}\left(\frac{1}{4 \pi}\right)^{2} p^{2} .
\end{gathered}
$$

(b) for $-g_{i j}\left(p_{\nu} \cdot p_{\tau}\right) N_{i} N_{j}^{*}$ term,

The same as Eq.(A11) but here for $M= \pm 1$, we have

$$
\begin{gathered}
-\frac{1}{m_{\tau} m_{\nu}} g_{i j}\left(p_{\nu} \cdot p_{\tau}\right) N_{i} N_{j}^{*}=\frac{1}{m_{\tau} m_{\nu}} \delta_{i j}\left(p_{\nu} \cdot p_{\tau}\right) N_{i} N_{j}^{*}=\frac{1}{m_{\tau} m_{\nu}} \sum_{\mu}\left(p_{\nu} \cdot p_{\tau}\right) N_{\mu} N_{\mu}^{*} \\
=\frac{1}{m_{\tau} m_{\nu}}\left(p_{\nu} \cdot p_{\tau}\right) \frac{1}{3}\left(\frac{1}{4 \pi}\right)^{2} \sum_{\mu} \mathcal{C}^{2}(111 ; M,-\mu, M-\mu) \\
=\frac{1}{m_{\tau} m_{\nu}}\left(p_{\nu} \cdot p_{\tau}\right) \frac{1}{3}\left(\frac{1}{4 \pi}\right)^{2}
\end{gathered}
$$

(c) for the $i \epsilon^{\alpha i \beta j} p_{\nu \alpha} p_{\tau \beta} N_{i} N_{j}^{*}$ term, $\alpha$ or $\beta$ must be zero.

First, if we take $\alpha=0$,

$$
\begin{gathered}
i \epsilon^{\alpha i \beta j} p_{\nu \alpha} p_{\tau \beta} N_{i} N_{j}^{*}=i E_{\nu} \epsilon^{i \beta j} p_{\tau \beta} N_{i} N_{j}^{*} \\
=-i E_{\nu} \epsilon^{3 i j} p N_{i} N_{j}^{*}=i i E_{\nu} p\left(N_{-} N_{-}-N_{+} N_{+}\right),
\end{gathered}
$$

then, Eq.(A20) becomes

$$
-E_{\nu} p \frac{1}{3}\left(\frac{1}{4 \pi}\right)^{2}\left[\mathcal{C}^{2}(111 ; M,+1, M+1)-\mathcal{C}^{2}(111 ; M,-1, M-1)\right] .
$$


Second, if we take $\beta=0$,

$$
\begin{gathered}
\\
i \epsilon^{\alpha i 0 j} p_{\nu \alpha} p_{\tau \beta} N_{i} N_{j}^{*}=i E_{\tau} \epsilon^{\alpha i j} p_{\nu \alpha} N_{i} N_{j}^{*} \\
=i E_{\tau} \epsilon^{3 i j} p N_{i} N_{j}^{*}=E_{\tau} p\left(N_{-} N_{-}-N_{+} N_{+}\right),
\end{gathered}
$$

then Eq.(A22) becomes

$$
E_{\tau} p \frac{1}{3}\left(\frac{1}{4 \pi}\right)^{2}\left[\mathcal{C}^{2}(111 ; M,+1, M+1)-\mathcal{C}^{2}(111 ; M,-1, M-1)\right] .
$$

then adding the results of Eq. (A21) and (A23), calculating the CGC explicitly we find including the factor $\frac{1}{m_{\tau} m_{\nu}}$

$$
\frac{1}{m_{\tau} m_{\nu}} i \epsilon^{\alpha i \beta j} p_{\nu \alpha} p_{\tau \beta} N_{i} N_{j}^{*}=\frac{1}{m_{\tau} m_{\nu}} \frac{1}{6}\left(\frac{1}{4 \pi}\right)^{2}\left(E_{\nu}-E_{\tau}\right) p M .
$$

Altogether, from Eqs. (A14), (A17), (A18), (A19), (A24), we obtain

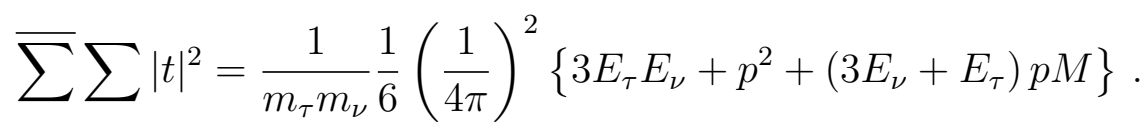

For the case of $\alpha \neq 1$, all we must do is to consider that now $N_{i}$ gets multiplied by $\alpha$ and we readily get the new equations.

[1] N. Isgur, C. Morningstar, and C. Reader, Phys. Rev. D 39, 1357 (1989).

[2] H. Kühn, F. Wagner, Nucl. Phys. B 236, 16 (1984).

[3] B. C. Barish, R. Stroynowski, Phys. Rept. 157, 1 (1988).

[4] M. Davier, A. Hócker, and Z. Q. Zhang, Rev. Mod. Phys. 78, 1043 (2006).

[5] M. Antonelli, D. M. Asner, D. Bauer et al, Phys. Rept. 494, 197 (2010).

[6] B. A. Li, Phys. Rev. D 52, 5165 (1995); 52, 5184 (1995).

[7] B. A. Li, Phys. Rev. D 55, 1436 (1997).

[8] D. Buskulic et al. (ALEPH Collaboration). Z. Phys. C 74, 263 (1997).

[9] (ALEPH Collaboration). Z. Phys. C 70, 579 (1996).

[10] R. Barate et al. (ALEPH Collaboration). Eur. Phys. J. C 1, 65 (1998).

[11] K. Inami et al. (Belle Collaboration). Phys. Lett. B 672, 209 (2009).

[12] K. Arms et al. (CLEO Collaboration), Phys. Rev. Lett. 94, 241802 (2005). 
[13] R. Barate et al. (ALEPH Collaboration). Eur. Phys. J. C 10, 1 (1999).

[14] B. Aubert et al. (The BABAR Collaboration), Phys. Rev. Lett. 100, 011801 (2008).

[15] M. K. Volkov, K. Nurlan and A. A. Pivovarov, JETP Lett. 106, 771 (2017).

[16] G. Lopez Castro and D. A. Lopez Falcon, Phys. Rev. D 54, 4400 (1996).

[17] M. K. Volkov, A. B. Arbuzov and D. G. Kostunin, Phys. Rev. D 86, 057301 (2012).

[18] L. R. Dai, R. Pavao, S. Sakai and E. Oset, arXiv:1805.04573 [hep-ph].

[19] L. Micu, Nucl. Phys. B 10, 521 (1969).

[20] A. Le Yaouanc, L. Oliver, Pène and J. C. Raynal, Phys. Rev. D 8, 2223 (1973).

[21] F. E. Close, An Introduction to Quark and Partons, Academic Press, 1979.

[22] W. H. Liang and E. Oset, Eur. Phys. J. C 78, 528 (2018).

[23] X. G. He and G. Valencia, Phys. Lett. B 779, 52 (2018).

[24] S. M. Boucenna, A. Celis, J. Fuentes-Martin, A. Vicente and J. Virto, Phys. Lett. B 760, 214 (2016).

[25] C. H. Chen, T. Nomura and H. Okada, Phys. Lett. B 774, 456 (2017).

[26] A. Crivellin, D. Müller, T. Ota, J. High Energy Phys. 1709, 040 (2017).

[27] D. Bečirević, I. Doršner, S. Fajfer, N. Košnik, D. A. Faroughy, O. Sumensari, arXiv:1806.05689

[28] M. Bordone, C. Cornella, J. Fuentes-Martin and G. Isidori, Phys. Lett. B 779, 317 (2018).

[29] N. Assad, B. Fornal and B. Grinstein, Phys. Lett. B 777, 324 (2018).

[30] M. Blanke, A. Crivellin, Phys. Rev. Lett. 121, 011801 (2018).

[31] X. G. He, G. Valencia, Phys. Lett. B 779, 52 (2018).

[32] M. Tanabashi et al. [Particle Data Group], Phys. Rev. D 98, 030001 (2018).

[33] K. Hagiwara, A. D. Martin and M. F. Wade, Phys. Lett. B 228, 144 (1989).

[34] R. Alonso, A. Kobach, and J. M. Camalich, Phys. Rev. D. 94, 094021 (2016).

[35] L. R. Dai and E. Oset, arXiv:1808.02876 [hep-ph].

[36] C. Itzykson and J. B. Zuber, Quantum Field Theory, Mecraw-Hill, 1980.

[37] F. Mandl and G. Shaw, Quantum Field Theory, John Wiley \& Sons, 1984

[38] X. G. He and G. Valencia, Phys. Rev. D 87014014 (2013).

[39] S. Schael et al. [ALEPH Collaboration], Phys. Rept. 421, 191 (2005).

[40] M. Wagner and S. Leupold, Phys. Rev. D 78, 053001 (2008).

[41] M. F. M. Lutz and E. E. Kolomeitsev, Nucl. Phys. A 730, 392 (2004).

[42] L. Roca, E. Oset and J. Singh, Phys. Rev. D 72, 014002 (2005). 
[43] Y. Zhou, X. L. Ren, H. X. Chen and L. S. Geng, Phys. Rev. D 90, 014020 (2014).

[44] I. Caprini, L. Lellouch and M. Neubert, Nucl. Phys. B 530, 153 (1998).

[45] S. Fajfer, J. F. Kamenik and I. Nisandzic, Phys. Rev. D 85, 094025 (2012).

[46] Q. Luo, D. R. Xu, Proceedings of the 9th International Particle Accelerator Conference (https://doi.org/10.18429/JACoW-IPAC2018-MOPML013).

[47] S. Eidelman, Nuclear and Particle Physics Proceedings, Vol. 260, 238 (2015).

[48] Joint Workshop of future tau-charm factory (https://indico.lal.in2p3.fr/event/4902/\#38-tauphysics-with-future-tau).

[49] E. Kou et al. [Belle II Collaboration], arXiv:1808.10567 [hep-ex].

[50] K. Adamczyk [Belle Collaboration], PoS CKM2016, 052 (2017) (https://pos.sissa.it/291/052).

[51] A. K. Alok, A. Datta, A. Dighe, M. Duraisamy, D. Ghosh and D. London, J. High Energy Phys. 1111, 121 (2011).

[52] K. Hagiwara, A. D. Martin and M. F. Wade, Phys. Lett. B 228, 144 (1989).

[53] X. G. He and G. Valencia, Phys. Rev. D 66, 013004 (2002); Erratum: [Phys. Rev. D 66, 079901 (2002)].

[54] U. Baur, T. Plehn and D. L. Rainwater, Phys. Rev. D 68, 033001 (2003).

[55] X. G. He and G. Valencia, Phys. Rev. D 87, 014014 (2013).

[56] A. K. Alok, D. Kumar, J. Kumar, S. Kumbhakar and S. U. Sankar, J. High Energy Phys. 1809, $152(2018)$.

[57] S. Bhattacharya, S. Nandi and S. Kumar Patra, arXiv:1805.08222 [hep-ph]. 